\title{
Spatial and temporal variation in the water quality of an urban drainage system in Ciudad del Carmen, México
}

\author{
M. del R. Barreto-Castro, A. Delgado-Estrella, \\ G. Acevedo-Olvera \& E. Nuñez-Lara \\ Facultad de Ciencias Naturales, \\ Universidad Autónoma del Carmen, México
}

\begin{abstract}
Anthropogenic activities generate pollutants that are often discharged, untreated, into bodies of water. The drainage system, known as the Caleta in Ciudad del Carmen, Mexico, is surrounded by an urban area and receives immense amounts of industrial and sewage discharge. Physicochemical analyses were conducted for the water and sediment samples taken along this drainage system to understand its hydrological dynamic and pollutant load. The drainage channel communicates with Terminos Lagoon, a Natural Protected Area, and the Gulf of Mexico. Water and sediment samples were taken at fifteen stations along the drainage channel during the three local seasons: northwinds (January); dry (May); and rainy (October). Physicochemical parameters $(\mathrm{pH}$, temperature, salinity, dissolved oxygen, and total dissolved solids), as well as biological oxygen demand (BOD 5 ), chemical oxygen demand (COD) and total suspended solids (TSS) were measured in the water samples. Heavy metals concentrations ( $\mathrm{Fe}, \mathrm{Cr}, \mathrm{Cu}, \mathrm{Zn}, \mathrm{Cd}$ and $\mathrm{Pb}$ ) were quantified in the sediment samples. No differences were observed in the physicochemical parameters between sample stations, although differences $(\mathrm{P}<0.05)$ were observed between seasons. Dissolved oxygen levels at all stations and in all seasons were near or below hypoxia levels $(<2.0 \mathrm{mg} / \mathrm{l})$. Biological oxygen demand and COD were highest during the rainy season, particularly near industrial effluent discharges. Heavy metals concentrations varied spatially, with higher levels nearest the drainage outlet and lower levels further inland. Lead and iron levels were extremely high, and all heavy metals concentrations far exceeded legal limits. The Caleta is clearly heavily impacted by discharge from the surrounding urban area containing pollutants generated by anthropogenic
\end{abstract}


activities. Any possible recovery of this drainage ecosystem will be contingent on treating and controlling any discharges into it.

Keywords: Terminos Lagoon, water quality, heavy metals, drainage, Ciudad del Carmen.

\section{Introduction}

Hydrology within Terminos Lagoon, Mexico, is influenced by the interaction between marine water entering from the Gulf of Mexico through inlets and freshwater influx from the Grijalva and Usumacinta river systems. Water circulation in the lagoon is most strongly affected by the east to west winds prevailing in the region (Graham et al. [1]). Ciudad del Carmen, Campeche, is located at the western tip of an island forming a barrier between the Gulf of Mexico and the lagoon. The city is a logistics center for petroleum extraction activities on offshore oil platforms in the southern Gulf. A drainage known as the Caleta runs east to west through the city, connecting to the lagoon at the west end. It is an ecologically important drainage, particularly because it interacts with the waters of the lagoon, which is classified as a natural protected area (NPA). Over time, pollution in the drainage has increased in response to anthropogenic activities. The primary pollutant sources are petroleum industry activities, which generate heavy metals and hydrocarbons, and agriculture, which contributes chemical run-off from fertilizers and pesticides. Residues from commercial and fishing activities also wash into the drainage. After years of rapid population growth in Ciudad del Carmen, it is now completely surrounded by urban area, and receives largely untreated sewage from the city's sewage system. Flora and fauna in the Caleta ecosystem have suffered extremely negative impacts from this pollution, and are considered pollutant bioindicators because they concentrate persistent, bioaccumulative toxic substances (Instituto Sindical de Trabajo, Ambiente y Salud [2]).

Its environmental matrix strongly influences the Caleta's hydrology. This determines material import and export rates within the system, and controls internal processes such as nutrient assimilation, organic matter storage, benthonic regeneration and nutrient release. Geological, physical, chemical, climatic and biological factors add their effects to anthropogenic activities, determining how the Caleta, and the greater Terminos Lagoon, functions.

The present study objective was to use water and sediment physicochemical parameters to characterize the Caleta drainage's hydrology and pollutant load during the three regional seasons.

\section{Methods}

\subsection{Study area}

The Caleta in Ciudad del Carmen is a surface drainage that connects to Terminos Lagoon at its western outlet (Figure 1). Located at the southern extreme of the Gulf of Mexico, in southeast Mexico $\left(91^{\circ} 15^{\prime}, 92^{\circ} 00^{\prime} \mathrm{W} ; 18^{\circ} 25^{\prime}\right.$ and $\left.19^{\circ} 00^{\prime} \mathrm{N}\right)$, 
the Terminos Lagoon measures approximately $70 \mathrm{~km}$ long and $28 \mathrm{~km}$ wide $\left(2500 \mathrm{~km}^{2}\right)$. This coastal lagoon system contains approximately fourteen minor coastal lagoons, and is encompassed by the "Gulf coastal plain" and "Yucatan Peninsula" physiographic provinces (Gutiérrez-Estrada and Castro del Río [3]). It is separated from the Gulf by Carmen Island (approx. $37.5 \times 3 \mathrm{~km}$ ), a barrier island (Tamayo [4]).

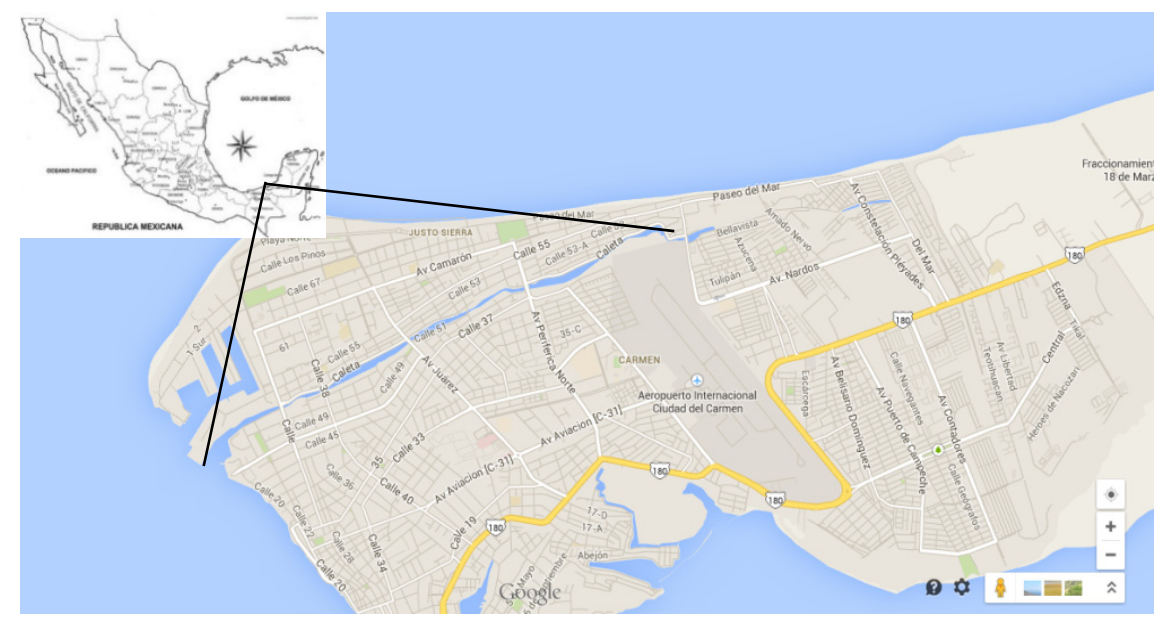

Figure 1: Study area: western end of Carmen Island, showing Ciudad del Carmen and the Caleta.

\subsection{Sample station locations}

Fifteen sample sites were placed along the $7.5 \mathrm{~km}$ length of the drainage.

Table 1: Sampling station locations.

\begin{tabular}{lll}
\hline Stations & \multicolumn{2}{c}{ Positions } \\
& Latitude & Longitude \\
\hline 1 & $\left(18^{\circ} 39^{\prime} 45.4^{\prime \prime}\right)$ & $\left(91^{\circ} 48^{\prime} 13.7^{\prime \prime}\right)$ \\
2 & $\left(18^{\circ} 39^{\prime} 47.8^{\prime \prime}\right)$ & $\left(91^{\circ} 48^{\prime} 23.5^{\prime \prime}\right)$ \\
3 & $\left(18^{\circ} 39^{\prime} 41.8^{\prime \prime}\right)$ & $\left(91^{\circ} 48^{\prime} 35.4^{\prime \prime}\right)$ \\
4 & $\left(18^{\circ} 39^{\prime} 345^{\prime \prime}\right)$ & $\left(91^{\circ} 48^{\prime} 50.5^{\prime \prime}\right)$ \\
5 & $\left(18^{\circ} 39^{\prime} 30.6^{\prime \prime}\right)$ & $\left(91^{\circ} 49^{\prime} 05.5^{\prime \prime}\right)$ \\
6 & $\left(18^{\circ} 39^{\prime} 24.1^{\prime \prime}\right)$ & $\left(91^{\circ} 49^{\prime} 21.3^{\prime \prime}\right)$ \\
7 & $\left(18^{\circ} 39^{\prime} 18.9^{\prime \prime}\right)$ & $\left(91^{\circ} 49^{\prime} 35.7^{\prime \prime}\right)$ \\
8 & $\left(18^{\circ} 39^{\prime} 10.3^{\prime \prime}\right)$ & $\left(91^{\circ} 49^{\prime} 48.0^{\prime \prime}\right)$ \\
9 & $\left(18^{\circ} 39^{\prime} 07.4^{\prime \prime}\right)$ & $\left(91^{\circ} 49^{\prime} 57.4^{\prime \prime}\right)$ \\
10 & $\left(18^{\circ} 39^{\prime} 01.2^{\prime \prime}\right)$ & $\left(91^{\circ} 50^{\prime} 07.7^{\prime \prime}\right)$ \\
11 & $\left(18^{\circ} 38^{\prime} 56.4^{\prime \prime}\right)$ & $\left(91^{\circ} 50^{\prime} 20.4^{\prime \prime}\right)$ \\
12 & $\left(18^{\circ} 38^{\prime} 50.1^{\prime \prime}\right)$ & $\left(91^{\circ} 50^{\prime} 35.9^{\prime \prime}\right)$ \\
13 & $\left(18^{\circ} 38^{\prime} 49.3^{\prime \prime}\right)$ & $\left(91^{\circ} 50^{\prime} 45.3^{\prime \prime}\right)$ \\
14 & $\left(18^{\circ} 38^{\prime} 43.2^{\prime \prime}\right)$ & $\left(91^{\circ} 50^{\prime} 37.6^{\prime \prime}\right)$ \\
15 & $\left(18^{\circ} 38^{\prime} 38.1^{\prime \prime}\right)$ & $\left(91^{\circ} 50^{\prime} 42.3^{\prime \prime}\right)$ \\
\hline
\end{tabular}




\subsection{Sample analysis}

Soil and water samples were collected during each of the three regional seasons: northwinds (January 2014); dry (May 2014); and rainy (October 2014). A multiparameter probe (Hanna HI $9828 \mathrm{PH} / \mathrm{ORP} / \mathrm{EC} / \mathrm{DO}$ ) was used to run triplicate, in situ analyses of $\mathrm{pH}$, temperature $\left({ }^{\circ} \mathrm{C}\right)$, salinity $(\%)$, dissolved oxygen $\left(\mathrm{O}_{2}\right)$ and total dissolved solids (TDS). Water samples were returned to the laboratory to calculate biological oxygen demand $\left(\mathrm{BOD}_{5}\right)$ using an electrometric test (NMX-AA-028-SCFI-2001), chemical oxygen demand (COD) with the closed flow test (NMX-AA-034-SCFI-2001) and total suspended solids (TSS) with the gravimetric test (NMX-AA-034-SCFI-2001).

Surface sediment samples were taken at each station using a $6 \times 6 \times 6$ " Ekman dredge. Presence of six heavy metals ( $\mathrm{Fe}, \mathrm{Cu}, \mathrm{Mn}, \mathrm{Zn}, \mathrm{Cd}$, and $\mathrm{Pb}$ ) was determined following EPA method 3050-B (US EPA [5], for acid digestion of sediments, muds and soils. Final metals concentrations were quantified using a double beam atomic absorption device (GBC-Avanta 3000, Flama) with an air-acetylene flame and deuterium background corrector. A calibration curve was prepared for each metal using recognized standard, analytical quality patterns within the analytical range (Table 2).

Table 2: Wavelength and application intervals.

\begin{tabular}{llccc}
\hline \multicolumn{1}{c}{ Element } & & Wavelength $(\mathrm{nm})$ & $\begin{array}{c}\text { Working range } \\
\mu \mathrm{gL}^{-1}\end{array}$ & Detection limit \\
\hline Iron & $(\mathrm{Fe})$ & 248.3 & $2.0-145$ & 0.1 \\
Chrome & $(\mathrm{Cr})$ & 357.9 & $0.1-50$ & 0.02 \\
Zinc & $(\mathrm{Zn})$ & 213.9 & $0.1-10$ & 0.2 \\
Cadmium & $(\mathrm{Cd})$ & 228.8 & $0.1-20$ & 0.02 \\
Lead & $(\mathrm{Pb})$ & 283.3 & $5.0-200$ & 0.1 \\
Copper & $(\mathrm{Cu})$ & 324.8 & $0.1-180$ & 0.07 \\
\hline
\end{tabular}

\subsection{Statistical analyses}

Data were interpreted using the Past ver. 3.5 statistical package. A completely random analysis of variance (ANOVA) was run to identify significant differences between sampling stations. Unequal variances were analyzed with the KruskallWallis (KW) non-parametric method in which the dependent variable was season and the significance level was $\mathrm{P} \leq 0.05$. Differences between the means were identified with a Duncan multiple range test (Duncan [6]), and standard error (SE) calculated by mean squared error.

\section{Results and discussion}

Average physicochemical variable data for the three seasons showed no differences in $\mathrm{pH}$ between stations $(\mathrm{KW}=21.17)$, with values ranging from 7.1 to 8.7 (Table 3). However, differences $(\mathrm{KW}=442.06, \mathrm{p} \leq 0.05)$ were observed in $\mathrm{pH}$ between seasons. Values were highest during the rainy season, particularly in stations nearer the drainage mouth. 
Table 3: Physicochemical parameters recorded by station.

\begin{tabular}{|c|c|c|c|c|c|c|c|c|c|c|c|}
\hline \multirow{2}{*}{ Station } & \multirow{2}{*}{ Season } & \multicolumn{2}{|c|}{$\mathrm{pH}$} & \multicolumn{2}{|c|}{$\mathrm{T}\left({ }^{\circ} \mathrm{C}\right)$} & \multicolumn{2}{|c|}{ Sal $(\% / 00)$} & \multicolumn{2}{|c|}{$\mathrm{O}_{2}(\mathrm{mg} / \mathrm{l})$} & \multicolumn{2}{|c|}{ TDS (mg/l) } \\
\hline & & Avg & SE & Avg & $\mathrm{SE}$ & Avg & $\mathrm{SE}$ & Avg & $\mathrm{SE}$ & Avg & SE \\
\hline \multirow{3}{*}{ SS 1} & Northwind & 7.9 & 0.05 & 23.94 & 1.25 & 3.63 & 0.94 & 1.50 & 0.01 & 1743 & 83.29 \\
\hline & Dry & 7.2 & 0.04 & 31.50 & 1.00 & 4.12 & 0.75 & 1.80 & 0.01 & 1985 & 66.63 \\
\hline & Rainy & 8.3 & 0.11 & 30.29 & 2.75 & 3.05 & 2.06 & 2.35 & 0.02 & 2245 & 183.23 \\
\hline \multirow{3}{*}{ SS 2} & Northwind & 7.4 & 0.03 & 23.65 & 0.75 & 5.32 & 0.56 & 1.93 & 0.01 & 1789 & 49.97 \\
\hline & Dry & 7.6 & 0.05 & 31.55 & 1.25 & 6.18 & 0.94 & 2.10 & 0.01 & 1973 & 83.29 \\
\hline & Rainy & 8.5 & 0.05 & 30.12 & 1.25 & 4.56 & 0.94 & 2.45 & 0.01 & 2376 & 83.29 \\
\hline \multirow{3}{*}{ SS 3} & Northwind & 7.1 & 0.12 & 23.69 & 3.00 & 5.45 & 2.25 & 1.98 & 0.02 & 1675 & 199.89 \\
\hline & Dry & 7.6 & 0.11 & 31.54 & 2.75 & 6.21 & 2.06 & 2.15 & 0.02 & 1950 & 183.23 \\
\hline & Rainy & 8.7 & 0.14 & 30.15 & 3.50 & 5.12 & 2.63 & 2.98 & 0.03 & 2419 & 233.20 \\
\hline \multirow{3}{*}{ SS 4} & Northwind & 7.9 & 0.13 & 23.78 & 3.25 & 6.74 & 2.44 & 2.17 & 0.02 & 1872 & 216.54 \\
\hline & Dry & 7.2 & 0.07 & 31.55 & 1.75 & 6.99 & 1.31 & 1.95 & 0.01 & 1975 & 116.60 \\
\hline & Rainy & 8.4 & 0.09 & 29.99 & 2.25 & 5.36 & 1.69 & 3.12 & 0.02 & 2489 & 149.91 \\
\hline \multirow{3}{*}{ SS 5} & Northwind & 7.3 & 0.06 & 23.30 & 1.50 & 5.95 & 1.13 & 2.41 & 0.01 & 1213 & 99.94 \\
\hline & Dry & 7.6 & 0.05 & 31.58 & 1.25 & 6.82 & 0.94 & 2.52 & 0.01 & 1325 & 83.29 \\
\hline & Rainy & 8.1 & 0.07 & 29.95 & 1.75 & 5.49 & 1.31 & 2.98 & 0.01 & 1828 & 116.60 \\
\hline \multirow{3}{*}{ SS 6} & Northwind & 7.2 & 0.08 & 23.55 & 2.00 & 5.99 & 1.50 & 1.98 & 0.01 & 2190 & 133.26 \\
\hline & Dry & 7.7 & 0.09 & 31.60 & 2.25 & 6.37 & 1.69 & 1.25 & 0.02 & 2135 & 149.91 \\
\hline & Rainy & 8.5 & 0.07 & 30.12 & 1.75 & 5.79 & 1.31 & 2.27 & 0.01 & 2318 & 116.60 \\
\hline \multirow{3}{*}{ SS 7} & Northwind & 7.9 & 0.08 & 23.78 & 2.00 & 6.78 & 1.50 & 2.13 & 0.01 & 2145 & 133.26 \\
\hline & Dry & 7.4 & 0.09 & 31.62 & 2.25 & 7.18 & 1.69 & 2.56 & 0.02 & 1958 & 149.91 \\
\hline & Rainy & 8.3 & 0.12 & 29.89 & 3.00 & 6.15 & 2.25 & 3.56 & 0.02 & 2456 & 199.89 \\
\hline \multirow{3}{*}{ SS 8} & Northwind & 7.1 & 0.08 & 23.65 & 2.00 & 9.85 & 1.50 & 2.19 & 0.01 & 1987 & 133.26 \\
\hline & Dry & 7.5 & 0.09 & 31.59 & 2.25 & 10.20 & 1.69 & 2.35 & 0.02 & 1875 & 149.91 \\
\hline & Rainy & 8.2 & 0.11 & 29.95 & 2.75 & 9.35 & 2.06 & 2.18 & 0.02 & 2531 & 183.23 \\
\hline \multirow{3}{*}{ SS 9} & Northwind & 7.6 & 0.05 & 23.69 & 1.25 & 11.95 & 0.94 & 2.99 & 0.01 & 1983 & 83.29 \\
\hline & Dry & 7.8 & 0.04 & 31.55 & 1.00 & 12.45 & 0.75 & 3.38 & 0.01 & 1770 & 66.63 \\
\hline & Rainy & 8.7 & 0.10 & 29.91 & 2.50 & 10.29 & 1.88 & 3.95 & 0.02 & 2431 & 166.57 \\
\hline
\end{tabular}


Table 3: Continued.

\begin{tabular}{|c|c|c|c|c|c|c|c|c|c|c|c|}
\hline \multirow{2}{*}{ Station } & \multirow{2}{*}{ eason } & \multicolumn{2}{|c|}{$\mathrm{pH}$} & \multicolumn{2}{|c|}{$\mathrm{T}\left({ }^{\circ} \mathrm{C}\right)$} & \multicolumn{2}{|c|}{ Sal $(\% / 00)$} & \multicolumn{2}{|c|}{$\mathrm{O}_{2}(\mathrm{mg} / \mathrm{l})$} & \multicolumn{2}{|c|}{ TDS (mg/l) } \\
\hline & & Avg & $\mathrm{SE}$ & Avg & SE & Avg & SE & Avg & SE & Avg & SE \\
\hline \multirow{3}{*}{ SS 10} & Northwind & 7.3 & 0.09 & 23.85 & 2.25 & 12.95 & 1.69 & 3.10 & 0.02 & 1863 & 149.91 \\
\hline & Dry & 7.8 & 0.08 & 31.68 & 2.00 & 13.83 & 1.50 & 3.45 & 0.01 & 1662 & 133.26 \\
\hline & Rainy & 8.5 & 0.13 & 29.87 & 3.25 & 12.56 & 2.44 & 4.10 & 0.02 & 2387 & 216.54 \\
\hline \multirow{3}{*}{ SS 11} & North & 7.9 & 0.05 & 23.79 & 1.25 & 15.12 & 0.94 & 3.99 & 0.01 & 1982 & 83.29 \\
\hline & Dry & 7.7 & 0.06 & 31.65 & 1.50 & 16.54 & 1.13 & 4.20 & 0.01 & 1532 & 99.94 \\
\hline & Rainy & 8.1 & 0.09 & 29.75 & 2.25 & 14.90 & 1.69 & 4.87 & 0.02 & 2764 & 149.91 \\
\hline \multirow{3}{*}{ SS 12} & Northwind & 7.4 & 0.09 & 23.81 & 2.25 & 17.35 & 1.69 & 4.10 & 0.02 & 2239 & 149.91 \\
\hline & Dry & 7.6 & 0.07 & 31.56 & 1.75 & 17.98 & 1.31 & 4.85 & 0.01 & 1459 & 116.60 \\
\hline & Rainy & 8.7 & 0.10 & 29.90 & 2.50 & 16.98 & 1.88 & 4.91 & 0.02 & 2691 & 166.57 \\
\hline \multirow{3}{*}{ SS 13} & Northwind & 7.9 & 0.08 & 23.80 & 2.00 & 24.10 & 1.50 & 4.95 & 0.01 & 1243 & 133.26 \\
\hline & Dry & 7.8 & 0.05 & 31.59 & 1.25 & 24.56 & 0.94 & 5.10 & 0.01 & 991 & 83.29 \\
\hline & Rainy & 8.7 & 0.09 & 29.92 & 2.25 & 23.45 & 1.69 & 5.15 & 0.02 & 1963 & 149.91 \\
\hline \multirow{3}{*}{ SS 14} & Northwind & 7.2 & 0.08 & 23.79 & 2.00 & 26.75 & 1.50 & 4.87 & 0.01 & 982 & 133.26 \\
\hline & Dry & 7.5 & 0.06 & 31.61 & 1.50 & 27.48 & 1.13 & 5.15 & 0.01 & 1012 & 99.94 \\
\hline & Rainy & 8.2 & 0.13 & 29.95 & 3.25 & 25.56 & 2.44 & 5.23 & 0.02 & 1873 & 216.54 \\
\hline \multirow{3}{*}{ SS 15} & Northwind & 8.1 & 0.07 & 23.80 & 1.75 & 29.56 & 1.31 & 4.99 & 0.01 & 1002 & 116.60 \\
\hline & Dry & 7.9 & 0.05 & 31.60 & 1.25 & 31.25 & 0.94 & 5.31 & 0.01 & 995 & 83.29 \\
\hline & Rainy & 8.3 & 0.12 & 29.99 & 3.00 & 27.89 & 2.25 & 5.59 & 0.02 & 1110 & 199.89 \\
\hline
\end{tabular}

Dissolved oxygen $\left(\mathrm{O}_{2}\right)$ exhibited no differences between stations, although all $\mathrm{O}_{2}$ values were near or below concentrations constituting hypoxia $(2.0 \mathrm{mg} / \mathrm{l})$. Levels were notably lower at stations near urban wastewater discharges. For instance, station 6 had the lowest overall value $(1.25 \mathrm{mg} / \mathrm{l})$, one clearly favored by the nearby discharge and decomposition of large quantities of organic matter.

Temperature varied according to season. It was lowest $\left(23^{\circ} \mathrm{C}\right)$ at almost all stations during the northwinds season, which is to be expected since cold north winds during this season lower air and water temperature. Temperatures were highest $\left(31.68^{\circ} \mathrm{C}\right)$ during the rainy and dry seasons in response to increased solar radiation.

As expected, salinity varied between stations $(\mathrm{KW}=51.26 \mathrm{p} \leq 0.05)$. Those nearest the drainage mouth, and therefore to the marine influence of the Gulf, had the highest values $(23.45-31.25 \%$ ). Between seasons, salinity levels at all stations were higher during the dry season due to a greater marine water influx (particularly at stations 10 to 15 ) in response to less freshwater outflow, higher solar radiation 
levels and consequent evaporation of the water column (Yañez-Arancibia and Day [7].

Total dissolved solids levels were highest during the rainy season due to wastewater discharge into the drainage (particularly at station 6).

The parameters $\mathrm{BOD}_{5}(\mathrm{mg} / \mathrm{l})$, TSS $(\mathrm{mg} / \mathrm{l})$, and COD $(\mathrm{mg} / \mathrm{l})$ are required by law NOM-001-SEMARNAT-1996 [8] in Mexico to establish permissible pollutant levels in wastewater discharge into type A bodies of water, specifically artificial reservoirs and natural lakes. These three variables are used to represent wastewater conditions because conventional treatments can mitigate them and/or fix them at established levels. Regulations in Mexico include levels determined by the National Water Commission (Comision Nacional de Agua - CNA) that must be met by the party responsible for the discharge, or for a specific receiving body of water (e.g. the Caleta), to comply with the National Water Law. Legal maximum concentrations are $150 \mathrm{mg} / \mathrm{BOD}_{5}, 125 \mathrm{mg} / \mathrm{l} \mathrm{SST}$, and $320 \mathrm{mg} / \mathrm{l} \mathrm{COD}$. Concentrations recorded for these three variables along the Caleta drainage significantly exceeded these legal maximum levels (Table 4). Clearly, this drainage receives immense amounts of polluting discharge from the surrounding urban area, as well as from the many companies in Ciudad del Carmen with permits allowing them to discharge wastewater into the Caleta.

Table 4: $\quad$ Physicochemical parameters (NOM-001-SEMARNAT-1996).

\begin{tabular}{|c|c|c|c|c|c|c|c|}
\hline \multirow{2}{*}{ Station } & \multirow{2}{*}{ Season } & \multicolumn{2}{|c|}{ TSS mg/l } & \multicolumn{2}{|c|}{$\mathrm{BOD}_{5} \mathrm{mg} / \mathrm{l}$} & \multicolumn{2}{|c|}{ COD mg/l } \\
\hline & & Avg & SE & Avg & SE & Avg & SE \\
\hline \multirow{3}{*}{ SS 1} & Northwind & 159 & 1.23 & 270 & 80 & 810 & 63.3 \\
\hline & Dry & 185 & 0.99 & 315 & 64 & 945 & 50.7 \\
\hline & Rainy & 256 & 2.71 & 435 & 176 & 1305 & 139.3 \\
\hline \multirow{3}{*}{ SS 2} & Northwind & 142 & 0.74 & 242 & 48 & 726 & 38.0 \\
\hline & Dry & 174 & 1.23 & 295 & 80 & 885 & 63.3 \\
\hline & Rainy & 230 & 1.23 & 391 & 80 & 1173 & 63.3 \\
\hline \multirow{3}{*}{ SS 3} & Northwind & 184 & 2.96 & 312 & 192 & 936 & 152.0 \\
\hline & Dry & 175 & 2.71 & 298 & 176 & 894 & 139.3 \\
\hline & Rainy & 235 & 3.45 & 399 & 224 & 1197 & 177.3 \\
\hline \multirow{3}{*}{ SS 4} & Northwind & 175 & 3.21 & 297 & 208 & 891 & 164.6 \\
\hline & Dry & 188 & 1.73 & 319 & 112 & 957 & 88.6 \\
\hline & Rainy & 306 & 2.22 & 521 & 144 & 1563 & 114.0 \\
\hline \multirow{3}{*}{ SS 5} & Northwind & 182 & 1.48 & 310 & 96 & 930 & 76.0 \\
\hline & Dry & 229 & 1.23 & 390 & 80 & 1170 & 63.3 \\
\hline & Rainy & 288 & 1.73 & 489 & 112 & 1467 & 88.6 \\
\hline
\end{tabular}


Table 4: Continued.

\begin{tabular}{|c|c|c|c|c|c|c|c|}
\hline \multirow{2}{*}{ Station } & \multirow{2}{*}{ Season } & \multicolumn{2}{|c|}{ SST $\mathrm{mg} / 1$} & \multicolumn{2}{|c|}{$\mathrm{BOD}_{5} \mathrm{mg} / \mathrm{l}$} & \multicolumn{2}{|c|}{ COD mg/l } \\
\hline & & Ave & E.E & Ave & E.E & Ave & E.E \\
\hline \multirow{3}{*}{ SS 6} & Northwind & 234 & 1.97 & 398 & 128 & 1194 & 101.3 \\
\hline & Dry & 257 & 2.22 & 437 & 144 & 1311 & 114.0 \\
\hline & Rainy & 361 & 1.73 & 614 & 112 & 1842 & 88.6 \\
\hline \multirow{3}{*}{ SS 7} & Northwind & 160 & 1.97 & 272 & 128 & 816 & 101.3 \\
\hline & Dry & 174 & 2.22 & 295 & 144 & 885 & 114.0 \\
\hline & Rainy & 217 & 2.96 & 369 & 192 & 1107 & 152.0 \\
\hline \multirow{3}{*}{ SS 8} & Northwind & 95 & 1.97 & 162 & 24 & 486 & 18.7 \\
\hline & Dry & 111 & 2.22 & 189 & 27 & 567 & 21.0 \\
\hline & Rainy & 154 & 2.71 & 261 & 33 & 783 & 25.7 \\
\hline \multirow{3}{*}{ SS 9} & Northwind & 85 & 1.23 & 145 & 15 & 436 & 11.7 \\
\hline & Dry & 104 & 0.99 & 177 & 12 & 531 & 9.4 \\
\hline & Rainy & 138 & 2.47 & 235 & 30 & 704 & 23.4 \\
\hline \multirow{3}{*}{ SS 10} & Northwind & 110 & 2.22 & 187 & 26.63 & 243 & 21.04 \\
\hline & Dry & 105 & 1.97 & 179 & 23.67 & 232 & 18.70 \\
\hline & Rainy & 141 & 3.21 & 239 & 38.47 & 311 & 30.39 \\
\hline \multirow{3}{*}{ SS 11} & Northwind & 105 & 1.23 & 178 & 14.80 & 232 & 11.69 \\
\hline & Dry & 113 & 1.48 & 191 & 17.76 & 249 & 14.03 \\
\hline & Rainy & 184 & 2.22 & 313 & 26.63 & 406 & 21.04 \\
\hline \multirow{3}{*}{ SS 12} & Northwind & 109 & 2.22 & 186 & 26.63 & 242 & 21.04 \\
\hline & Dry & 138 & 1.73 & 234 & 20.71 & 304 & 16.36 \\
\hline & Rainy & 173 & 2.47 & 293 & 29.59 & 381 & 23.38 \\
\hline \multirow{3}{*}{ SS 13} & Northwind & 140 & 1.97 & 239 & 23.67 & 310 & 18.70 \\
\hline & Dry & 154 & 1.23 & 262 & 14.80 & 341 & 11.69 \\
\hline & Rainy & 217 & 2.22 & 368 & 26.63 & 479 & 21.04 \\
\hline \multirow{3}{*}{ SS 14} & Northwind & 96 & 1.97 & 163 & 23.67 & 212 & 18.70 \\
\hline & Dry & 104 & 1.48 & 177 & 17.76 & 230 & 14.03 \\
\hline & Rainy & 130 & 3.21 & 221 & 38.47 & 288 & 30.39 \\
\hline \multirow{3}{*}{ SS 15} & Northwind & 195 & 1.73 & 332 & 20.71 & 431 & 16.36 \\
\hline & Dry & 214 & 1.23 & 364 & 14.80 & 473 & 11.69 \\
\hline & Rainy & 298 & 2.96 & 369 & 35.51 & 480 & 28.05 \\
\hline
\end{tabular}


Heavy metals concentrations exhibited a value gradient with the highest values nearest the drainage outlet (Table 5). This suggests that these metals most probably originate in discharge from areas surrounding the drainage, direct influents into it, and rainfall wash from the urban area. Lead and iron had significantly high levels. The former is an indicator of anthropogenic activity and the latter is a mobile element. Both can form precipitates with other heavy metals and are indicative of

Table 5: Heavy metal values in sediments, Caleta drainage, Ciudad del Carmen, Campeche.

\begin{tabular}{|c|c|c|c|c|c|c|c|}
\hline \multirow{2}{*}{ Station } & \multirow{2}{*}{ Season } & \multicolumn{6}{|c|}{ Heavy metals $(\mu \mathrm{g} / \mathrm{g})$} \\
\hline & & $\mathrm{Fe}$ & $\mathrm{Cr}$ & $\mathrm{Cu}$ & $\mathrm{Zn}$ & $\mathrm{Cd}$ & $\mathrm{Pb}$ \\
\hline \multirow{3}{*}{ SS 1} & Northwind & 66.5 & 21.45 & 1.38 & 0.32 & 1.76 & 95.00 \\
\hline & Dry & 50.25 & 16.21 & 1.24 & 0.25 & 1.59 & 71.79 \\
\hline & Rainy & 71.4 & 23.03 & 2.00 & 1.78 & 2.57 & 91.54 \\
\hline \multirow{3}{*}{ SS 2} & Northwind & 73.15 & 23.60 & 1.51 & 0.35 & 1.94 & 104.50 \\
\hline & Dry & 55.28 & 17.83 & 1.36 & 0.27 & 1.75 & 78.96 \\
\hline & Rainy & 78.54 & 25.34 & 2.20 & 1.96 & 2.82 & 100.69 \\
\hline \multirow{3}{*}{ SS 3} & Northwind & 79.80 & 25.74 & 1.65 & 0.38 & 2.12 & 114.00 \\
\hline & Dry & 60.30 & 19.45 & 1.48 & 0.30 & 1.90 & 86.14 \\
\hline & Rainy & 85.68 & 27.64 & 2.40 & 2.14 & 3.08 & 109.85 \\
\hline \multirow{3}{*}{ SS 4} & Northwind & 83.13 & 26.81 & 1.72 & 0.40 & 2.20 & 118.75 \\
\hline & Dry & 62.81 & 20.26 & 1.55 & 0.31 & 1.98 & 89.73 \\
\hline & Rainy & 89.25 & 28.79 & 2.50 & 2.23 & 3.21 & 114.42 \\
\hline \multirow{3}{*}{ SS 5} & Northwind & 86.45 & 27.89 & 1.79 & 0.41 & 2.29 & 123.50 \\
\hline & Dry & 65.33 & 21.07 & 1.61 & 0.32 & 2.06 & 93.32 \\
\hline & Rainy & 92.82 & 29.94 & 2.60 & 2.31 & 3.34 & 119.00 \\
\hline \multirow{3}{*}{ SS 6} & Northwind & 89.78 & 28.96 & 1.86 & 0.43 & 2.38 & 128.25 \\
\hline & Dry & 67.84 & 21.88 & 1.67 & 0.33 & 2.14 & 96.91 \\
\hline & Rainy & 96.39 & 31.09 & 2.70 & 2.40 & 3.47 & 123.58 \\
\hline \multirow{3}{*}{ SS 7} & Northwind & 99.75 & 32.18 & 2.06 & 0.48 & 2.64 & 142.50 \\
\hline & Dry & 75.38 & 24.31 & 1.86 & 0.37 & 2.38 & 107.68 \\
\hline & Rainy & 107.10 & 34.55 & 3.00 & 2.67 & 3.85 & 137.31 \\
\hline \multirow{3}{*}{ SS 8} & Northwind & 103.08 & 33.25 & 2.13 & 0.49 & 2.73 & 147.25 \\
\hline & Dry & 77.89 & 25.13 & 1.92 & 0.38 & 2.46 & 111.27 \\
\hline & Rainy & 110.67 & 35.70 & 3.10 & 2.76 & 3.98 & 141.88 \\
\hline \multirow{3}{*}{ SS 9} & Northwind & 106.40 & 34.32 & 2.20 & 0.51 & 2.82 & 152.00 \\
\hline & Dry & 80.40 & 25.94 & 1.98 & 0.40 & 2.54 & 114.86 \\
\hline & Rainy & 114.24 & 36.85 & 3.20 & 2.85 & 4.11 & 146.46 \\
\hline
\end{tabular}


Table 5: Continued.

\begin{tabular}{|c|c|c|c|c|c|c|c|}
\hline \multirow{2}{*}{ Station } & \multirow{2}{*}{ Season } & \multicolumn{6}{|c|}{ Heavy metals $(\mu \mathrm{g} / \mathrm{g})$} \\
\hline & & $\mathrm{Fe}$ & $\mathrm{Cr}$ & $\mathrm{Cu}$ & $\mathrm{Zn}$ & $\mathrm{Cd}$ & $\mathrm{Pb}$ \\
\hline \multirow{3}{*}{ SS 10} & Northwind & 109.73 & 35.40 & 2.27 & 0.53 & 2.91 & 156.75 \\
\hline & Dry & 82.91 & 26.75 & 2.04 & 0.41 & 2.62 & 118.45 \\
\hline & Rainy & 117.81 & 38.00 & 3.30 & 2.94 & 4.24 & 151.04 \\
\hline \multirow{3}{*}{ SS 11} & Northwind & 113.05 & 36.47 & 2.34 & 0.54 & 3.00 & 161.50 \\
\hline & Dry & 85.43 & 27.56 & 2.10 & 0.42 & 2.70 & 122.04 \\
\hline & Rainy & 121.38 & 39.15 & 3.40 & 3.03 & 4.37 & 155.62 \\
\hline \multirow{3}{*}{ SS 12} & Northwind & 116.38 & 37.54 & 2.41 & 0.56 & 3.09 & 166.25 \\
\hline & Dry & 87.94 & 28.37 & 2.17 & 0.43 & 2.78 & 125.63 \\
\hline & Rainy & 124.95 & 40.31 & 3.50 & 3.12 & 4.49 & 160.19 \\
\hline \multirow{3}{*}{ SS 13} & Northwind & 119.70 & 38.61 & 2.48 & 0.57 & 3.17 & 171.00 \\
\hline & Dry & 90.45 & 29.18 & 2.23 & 0.45 & 2.86 & 129.21 \\
\hline & Rainy & 128.52 & 41.46 & 3.61 & 3.20 & 4.62 & 164.77 \\
\hline \multirow{3}{*}{ SS 14} & Northwind & 123.03 & 39.69 & 2.54 & 0.59 & 3.26 & 175.75 \\
\hline & Dry & 92.96 & 29.99 & 2.29 & 0.46 & 2.93 & 132.80 \\
\hline & Rainy & 132.09 & 42.61 & 3.71 & 3.29 & 4.75 & 169.35 \\
\hline \multirow{3}{*}{ SS 15} & Northwind & 126.35 & 40.76 & 2.61 & 0.60 & 3.35 & 180.50 \\
\hline & Dry & 95.48 & 30.80 & 2.35 & 0.47 & 3.01 & 136.39 \\
\hline & Rainy & 135.66 & 43.76 & 3.81 & 3.38 & 4.88 & 173.92 \\
\hline \multirow{3}{*}{ Mean } & Northwind & 99.75 & 32.18 & 2.06 & 0.48 & 2.64 & 142.50 \\
\hline & Dry & 75.38 & 24.31 & 1.86 & 2.34 & 2.38 & 107.68 \\
\hline & Rainy & 107.10 & 34.55 & 3.00 & 4.56 & 3.85 & 137.31 \\
\hline \multirow{3}{*}{ Std. Dev. } & Northwind & 5.87 & 1.89 & 0.12 & 0.03 & 0.16 & 8.38 \\
\hline & Dry & 4.43 & 1.43 & 0.11 & 0.14 & 0.14 & 6.33 \\
\hline & Rainy & 6.30 & 2.03 & 0.18 & 0.27 & 0.23 & 8.08 \\
\hline
\end{tabular}

general eutrophication processes (Aulio [9]). These metals are widely used by a number of companies in the city and its environs providing services to the state petroleum company, Petróleos Mexicanos (Pemex). Bioavailable metals such as zinc and cadmium exhibited higher concentrations near the drainage outlet during the rainy season than previously reported levels (Aguilar-Ucán et al. [10]). Heavy metal concentrations were also higher during the northwind season than during the dry season, mainly because high winds lead to sediment suspension which makes metals available for biota and allows them to circulate throughout the drainage. As mentioned, overall heavy metals concentrations and bioavailable metals concentrations far exceeded legal limits; concentrations this high are probably due largely to anthropogenic sources within Ciudad del Carmen. 


\section{Conclusions}

The present results clearly indicate that the Caleta drainage in Ciudad del Carmen, Mexico, receives an immense pollutant load from the surrounding urban area. Pollutant accumulation was highest in sediments, which could have detrimental long-term effects. When combined with environmental and seasonal conditions in the area, these pollutants have created extremely unfavorable conditions for the area's biota. Spatial and temporal variation within the Caleta drainage were determined by mixing processes caused by a number of factors: marine water from the Gulf; freshwater influx from rivers; pollutant discharges from industry and irregular human settlements; and internal circulation patterns caused by seasonal weather, particularly northwinds. Understanding how these factors interact is vital to quantifying the impacts suffered by this drainage. Influence from Terminos Lagoon and the Gulf of Mexico slightly mitigate the impact of anthropogenic pollution in the Caleta, but only control and treatment of sewage and industrial discharge will stop further deterioration of this ecosystem.

\section{Acknowledgement}

We wish to thank de PROFOCIE 2014 for the financial support for this paper.

\section{References}

[1] Graham, D.S., J.P. Daniels, J.M. Hill \& J. W. Day, Jr. A preliminary model of the circulation of Laguna de Terminos, Campeche, Mexico. Anales Inst. Ci. Mar limnol. Univ. Nac. Autón. Méx. 8(1): 51-62, 1981.

[2] Instituto Sindical de Trabajo, Ambiente y Salud. Sustancias tóxicas, persistentes y bioacumulativas (TPB). Recuperado de: http://www.istas.ccoo.es/ecoinformas/index.as p?idpagina $=613.2005$

[3] Gutiérrez-Estrada, M. \& A. Castro del Río. Origen y desarro geologico de la Laguna de Términos. In: A. Yáñez Arancibia \& J.W. Day, Jr. (eds). Ecologia de los ecosistemas costeros del sur del golfo de México: La región de la Laguna de Terminos. Universidad Nacional Autónoma de México. pp. 89-110, 1988.

[4] Tamayo, J.L. Geografía general de México. v. 1: Geografía física. Instituto Mexicano de Investigaciones Económicas, México, 562 pp., 1949.

[5] US EPA. Method 3050-B, Acid digestion of sediments, sludges and soils. SW-846. (Chapter 3): Metallic analyses, 1, A, Part I, Test methods for evaluating solid waste. United States Environmental Protection Agency, Washington DC, 1986.

[6] Duncan, D.B. Multiple Range and multiple $F$ test. Biometrics. 11: 1-42, 1955.

[7] Yañez-Arancibia, A., \& J.W. Day. Ecosystem Functioning: The Basis for Sustainable Management on Terminos Lagoon, Campeche México. Jalapa, Veracruz, México: Institute of Ecology A.C. 2005. 
[8] Diario Oficial de la Federación de fecha 23 de abril de 2003. NOM-001SEMARNAT-1996.

[9] Aulio K. Heavy metals in the Green algae Cladophora glomerata as related to shore types in the Archipielago Sea, SW Finland. Mar. Poll. Bull., 60: 1625-1630, 1983.

[10] Aguilar-Ucán C.A., C. Montalvo-Romero M.A Ramírez-Elias \& C. González Barrera. Metales pesados en sedimentos del arroyo "La Caleta" de Ciudad del Carmen, Campeche, México: estudio preliminar. Revista Latinoamericana de Recursos Naturales 5(3): 232-237, 2009. 